\title{
Suburban buffers as key areas in a sustainable city
}

\author{
K. Barełkowska \& L. Chlasta \\ University of Technology and Life Sciences in Bydgoszcz, Poland
}

\begin{abstract}
Harmonious development, management and thriving of urban space depends much on places of seemingly little importance - modest suburban areas of housing, industrial zones, mixed peripheral developments, but zooming in to the point of analyzing the urban detail, also the areas that bring those zones together - the urban buffers. The urban buffers may be defined as seams between areas of diverse urban functions, particularly those of contradicting functions.

The dynamic development processes often occurring in peripheral zones of cities are subject to multiple social, political and economic pressure factors and diminish the rationality of planning and spatial management. On one hand, there is a classic process of urban sprawl, well described in the literature and resulting mostly in housing pouring out of timely limits of urban structures. On the other hand, there are larger infrastructural or industrial developments with their primary assumption on being settled in well communicated but cheap land plots. This kind of mixture results in creating interweaved fabric of many functions and their areas of influence overlapping and producing conflicting zones.

This paper will attempt to diagnose and systematize buffer areas as well as confront ways the conflicts manifest with possible planning and design solutions aimed at weakening negative effects or eliminating those effects.

Keywords: buffer zone, transitional zone, incompatibility areas, matrix of buffers.
\end{abstract}

\section{Introduction}

Modern cities have a number of problems. Residents fled and continue to flee before them out of the city to the suburbs. Suburbs, which are almost as long as the city itself, at the time was the area that is a highly organized buffer between city life and nature and the countryside. At the same time it was space which 
eventually subject turned on or absorbed by the city [1]. Contemporary suburbs, which grew to the size of comparable cities, cumulate a number of old problems of urban areas, but also produce new ones, specific to their satellite location, generated by diverse factors, including development of technology and the expansion of technology.

Suburbs are no longer just for residential areas or small manufacturing and trading distant from the city center. They are also exclusive industrial areas, parks so characteristic of zoning functioning in spatial planning no major obstacles to the 80s. Low land prices have become just as attractive for people looking for a place to live and for the industry. In the wake of them also trade and other services entered a phase of migration from urban centers. In addition, the development of both the urban and surrounding satellite towns and cities make less and less urban planning for greenfield and more and more transformation parameters of existing urbanized environment. Therefore, the suburbs have become, just as city areas, a conglomerate of areas built and used in a various way.

The problem deepens if we take into account the fact that the spill to the suburbs ofnew constructions imposed on areas that had already been used, particularly for agriculture, and consequently had their structure and infrastructure. As a result of such mixing and interleaving, there is a formation of a tissue with many functions and areas of influence. This situation can have a positive impact on the neighborhood by promoting mutual development. More often, however, there is the phenomenon of a negative impact.

Therefore, there must be a kind of protection applied that would protect one area against the other one, or that would even save both areas against various factors that can be found on either of them.

The buffer zone is an attempt to solve the problem of incompatibility of continuous use of the various areas. It is assumed that it would alleviate the occurrence of a conflict between certain areas. Its primary mission is to protect and to level negative environmental influences. This can be done by either integrating the areas or, in some cases, separating them.

Therefore, the buffer zone is located around the peripheries of the areas or it may become an area that connects, penetrates two or more protected area in order to increase the rate of their growth and the efficiency of protection.

There are two main types of buffers. The first one - "the functional buffer" derived from the mainstream land, in its typical form of historical (zoning) functions. The second one - "the landscape buffer" - has appeared relatively recently.

The aim of the two buffers is the same - namely, to alleviate or even to prevent the harmful effects of one type of land use on the neighbouring land. The landscaped buffer operates on a different principle, it is a kind of a designed device in the form of greenery. It hides or camouflages undesirable views - the effect of this, unfortunately, is difficult to measure in quantitative terms, and its success lies in the realm of psychology and aesthetics-and serves as an acoustic screen. 


\section{Incompatibility}

In this paper we will look particularly at the "functional buffer" which should take into account a number of factors affecting the incompatibility of an adjacent land. The purpose of this narrowing is associated with the subordinate role of investigations presented here in relation to the decision-making process, early planning simulation, using the FAST application (developed by the team: Barełkowski, Wardeski, Janusz, Barełkowska, Chlasta). FAST is a simple utility computing which aims to promote sustainable planning at local and regional level. Its main objective was to create a framework for the evaluation of certain parameters such as the average level of demographic, demand for media and investment rates [2].

The concept of compliance is very important in zoning and is one of the main reasons for dividing types of use. In determining compliance, one must assess factors such as property values, traffic conflict - mixing pedestrians and vehicular traffic, the phenomena that affect the senses (noise, light, odor), aesthetic, psychological factors as well as the buildings and their volume.

From the point of view of residential areas, non-use is measured by the following factors: (1) a threat to people or property (e.g. fire, explosion, corrosive fumes threat, the threat of moving cars and trucks, railways, aircraft traffic); (2) a threat to health, convenience and comfort (e.g. excessive pollution, odors, noise (including traffic noise), vibration, glare at night, industrial waste, garbage, barriers to access of light and air, overcrowding); (3) a threat to morality (such as gambling, etc.); (4) other various factors (such as aesthetics, psychological and physical degradation of the neighborhood, including the appearance of land and buildings, trademarks, decrease in the homogeneity of the neighborhood, encroachment of parking for customers of commercial services on residential streets, increasing vehicular traffic caused by industrial and commercial, etc.).

From the point of view of commercialism, non-uses and buildings can be measured by the "incompatibility of economic factors," such as the use of land and buildings, which interrupt the flow of pedestrian traffic in the area of trade. Such gaps are created by (a) 'dead spots', where customers lose interest, (b) driveways and other physical breaks in the pavement, (c) traffic intersection, both for vehicles and pedestrians, and (d) areas with threat: noise, odors, ugliness, and other unpleasant traits [3].

Similarly today, the industry recognizes the new design standards of modern factories and planned industrial districts.

Compatibility or incompatibility depends on the characteristics and performance of various applications and the conditions in which they operate. Therefore, the design of a buffer zone well-fulfilling its role of needs to be examined: What does the buffer intend to achieve? What are the conditions for its implementation? Do the individual provisions of the zone meet these conditions?

As it has been written before, the buffer is supposed to protect. Due to the multitude of sites occurring in the cities and their suburbs, it is not possible to adopt a universal "compatible frontier zone" and universal buffer zone. Buffers used in different areas will always look different. They can be analyzed for a 
variety of issues. Although the variables are many, they are not random - they must be combined into an organic whole [4].

\section{Materials}

Preparation of a matrix of buffers ca help in the creation of buffers. It has already been created for the development of spatial management Rokietnica (Poland) in a simplified form [5], and now for the spatial management Oborniki (Poland) - in the expanded version. For the purposes of this paper, it has been simplified. It takes into account only the basic areas located on the area covered by the development and selected factors affecting the incompatibility factors. The following description is to present a method for compiling, which can also be used in other planning publications.

To create a matrix, it is necessary to follow the steps preceding it:

1) Identification of lands in a given area based on the nature and type of building.

2) Classification of certain areas due to the nature of the basic function: residential $(\mathrm{R})$, building service and commercial $(\mathrm{U})$, industrial $(\mathrm{P})$, or other (X-variable depending on the specific function).

3) Statements of position parameters determining the nature of the different areas: building area, building height, etc.

4) Listing the negative factors generated at different sites.

5) Preparation of the matrix.

\subsection{Identify areas}

Determination of lands in a given area based on the nature and type of building for the area used in the discussion of the example is as follows:

- $\mathrm{MJ}$ - the area of single-family housing, only free-standing and a twin;

- $\mathrm{MZ}$ - compact residential area, in the area of single-family houses or multifamily, townhouses, as well as the central character, which means a continuous building frontage of varying heights and varying intensity;

- $\mathrm{MW}$ - the area of multi-family housing;

- $\mathrm{U}$ - area of service, including building area of different services, that is all the fields of activity classified in separate regulations as a service, with the exception of trade services implemented on a net area of more than $2000 \mathrm{~m}^{2}$;

- UD - area of fine services and small trade, occurring only in conjunction with other explanations, and for small trade - trade services realized on the sales area not exceeding $400 \mathrm{~m}^{2}$;

- $\mathrm{UC}$ - area of services trade, while allowing trade services implemented on both the sales area not exceeding $2000 \mathrm{~m}^{2}$ and $2000 \mathrm{~m}^{2}$ in excess;

- US - area of sport and recreation services;

- UA - area of administrative services, office, social and municipal services;

- UM - area of health services and social services;

- UN - area of services, education and science;

- $\mathrm{P}-$ area of the industry. 
These types of sites here relate to the most important category, although the constructed matrix covers a wider range of types, with specific profiles that influence later on the shape and individualization of the urban fabric. The matrix is an open structure, arguing for the transition from the research process in Rokietnica to the process implemented in Oborniki, it must be possible to introduce new types and modify existing parameters stored in the matrix, where appropriate to the local conditions. Studying the interaction between areas with different functions dominant does not change the principle but it allows to take a better account of the specific nature of local space, scale, relationships, geographical habitat and unique cultural elements. Depending on the situation, a number of very unusual terms of function areas may appear; for example airports, seaports, river areas, UNESCO World Heritage Site.

\subsection{Classification areas}

Classification of certain areas due to the nature of the basic function: residential $(\mathrm{R})$, building service and commercial $(\mathrm{U})$, industrial $(\mathrm{P})$, or other (X-variable depending on the specific function). It is important not to generalize specific functions, which in reality are rarely compatible with each other. For example, the classification of this looks as follows (see Table 1). The residential areas include: single-family housing $(\mathrm{MJ})$, dense residential development (MZ) and apartment housing (MW).

Service- trade areas include: service development (U), development of small trade and small services (UD,) development of large-services (UC) and administrative services buildings, offices, social and municipal services (UA). Areas of building industry $(\mathrm{P})$, like other areas such as: sports and recreation services (US), health services building areas (UM) and building services, education and science (UN) were given their own categories.

\subsection{Parameters of the building}

Position statement of the parameters which determine the nature of the different areas. For the purposes of the study, the indicator of the total square feet of a building divided by the total square feet of the lot the building is located on (FAR) has been chosen. FAR is used by local governments in zoning codes [6]. Higher FAR seems to indicate a more urban (dense) development.

Data is presented in a range format. This is due to the fact that this ratio is not constant and is set in a certain specific ranges. This factor may be replaced by juxtaposition of building parameters. The data is presented in Table 1.

\subsection{Negative factors}

Statement of negative factors generated at different sites. Only a few of factors such as noise, pollution and odor have been selected for the discussed area. They problems most often mentioned as a nuisance by city dwellers and they arise from the usage of the site. They can stem not only from the use of machinery and equipment but also the increased vehicular or pedestrian traffic. Table 1 presents 
a list of areas, including negative factors generated by them. The table does not indicate the level of generation of the negative factors but merely states the possibility of its occurrence in a given area. The occurrence is indicated only if there is a possibility of a large disturbance. As a result, it has been proved that the high risk of unpleasant odors is present in industrial areas (P). The issue of pollution is similar. The areas where the pollution can occur in the burdensome form are industrial areas (P) and the areas of medical services (UM). It is assumed that the noise does not occur in areas of single-family housing (MJ) and in the areas of sport and recreation services (US).

Table 1: Statement of factors.

\begin{tabular}{|c|c|c|c|c|c|c|c|c|c|c|c|}
\hline & $\mathrm{F}$ & $\mathrm{n}$ & o & $\mathrm{p}$ & FAR & 0 & 1 & 2 & 3 & 4 & 5 \\
\hline MJ & $\mathrm{r}$ & 0 & 0 & 0 & $0-1.0$ & & & & & & \\
\hline $\mathrm{MZ}$ & $\mathrm{r}$ & 1 & 0 & 0 & $1.0-6.6$ & & & & & & \\
\hline MW & $\mathrm{r}$ & 1 & 0 & 0 & $1.7-10.0$ & & & & & & \\
\hline $\mathrm{U}$ & $\mathrm{u}$ & 1 & 0 & 0 & $1.2-5$ & & & & & & \\
\hline UD & $\mathrm{u}$ & 1 & 0 & 0 & $0-1.2$ & & & & & & \\
\hline UC & $\mathrm{u}$ & 1 & 0 & 0 & $1.2-5$ & & & & & & \\
\hline US & $\mathrm{s}$ & 0 & 0 & 0 & $0-1.2$ & & & & & & \\
\hline UA & $\mathrm{u}$ & 1 & 0 & 0 & $>1.0$ & & & & & & \\
\hline UM & $\mathrm{h}$ & 1 & 0 & 0 & $0.2-3$ & & & & & & \\
\hline UN & $\mathrm{n}$ & 1 & 0 & 0 & $0.2-3$ & & & & & & \\
\hline$P$ & $\mathrm{p}$ & 1 & 1 & 1 & $1.2-3$ & & & & & & \\
\hline
\end{tabular}

Legend:

$\mathrm{F}$ - basic functions: $\mathrm{r}$ - housing; $\mathrm{u}$ - service and commercial; $\mathrm{p}$ - industrial; $\mathrm{s}$ - sports and recreation; $\mathrm{h}$ - health service; $\mathrm{n}$ - science and education; $\mathrm{n}$ - noise; $\mathrm{o}$ - odor; $\mathrm{p}$ - pollution; FAR - Floor Area Ratio - The total square feet of a building divided by the total square feet of the lot the building is located on.

\section{Matrix of buffers}

After analyzing fundamental incompatibility between the ring-fenced areas, the next step in the analysis is to attempt to determine the strength of the interaction. What can be helpful is a table which determines the factors that should be considered when designing a buffer. It must be, however, emphasized that the value relating to the function (f) is introduced only in the case when the functions are different for the two areas. The value for factors such as noise (n), pollution (p) and odor (o) is introduced always when at least one factor of the given ones appears in the area. In the situation when the function of the two sites is the same - there is no conflict function. It is assumed that if in both areas there is the same negative factor, it will not be a nuisance.

FAR rate issue is as follows: If the range of the coefficients corresponds to a certain degree, or range for this ratio is within the scope of one of the following 
just before or just after, it is referred to as a symbol table ((d)). It is assumed therefore that in certain situations the buffer will have to take account of this factor. If between FAR factor range of one area and the factor range of the other area there is no correspondence or the ranges do not overlap, a symbol (d) is used in the table. And then the buffer need to take this factor into account. All the factors are presented in Table 2.

Table 2: Matrix of buffers.

\begin{tabular}{|l|l|l|l|l|l|l|l|l|l|l|l|}
\hline & MJ & MZ & MW & U & UD & UC & US & UA & UM & UN & P \\
\hline MJ & x & B & C & E & D & E & D & D & F & D & G \\
\hline MZ & B & x & A & D & D & D & D & D & F & D & G \\
\hline MW & C & A & x & D & E & D & D & D & F & D & G \\
\hline U & E & D & D & x & A & A & D & A & F & D & G \\
\hline UD & D & D & E & A & x & A & D & A & F & D & G \\
\hline UC & E & D & D & A & A & x & D & A & F & D & G \\
\hline US & D & D & D & D & D & D & x & D & F & D & G \\
\hline UA & D & D & D & A & A & A & D & x & F & D & G \\
\hline UM & F & F & F & F & F & F & F & F & x & F & G \\
\hline UN & D & D & D & D & D & D & D & D & F & x & G \\
\hline P & G & G & G & G & G & G & G & G & G & G & x \\
\hline
\end{tabular}

Legend:
\begin{tabular}{|c|c}
\hline (d) & A \\
\cline { 1 - 1 } n(d) & B \\
\cline { 1 - 1 } nd & C \\
\cline { 1 - 1 } n(d)f & D \\
\cline { 1 - 1 } ndf & E \\
\cline { 1 - 1 }$(\mathrm{npd}) \mathrm{f}$ & $\mathrm{F}$ \\
\cline { 1 - 1 } nop(d)f & G
\end{tabular}

From the table it can be seen that the area may have 7 basic types of buffers.

Buffer ((d)) is designed to potentially mitigate two different types of building parameters specific to each of them. If there is no difference in the parameters of development, then there is no need to create a buffer.

Buffer (n(d)) has the task to level off potential of two different types of building parameters and to protect one of the areas against the noise generated in the second area. If there is no need for leveling the building parameters, a buffer will act as a protection against the noise.

Buffer (nd) has the task, apart from y leveling off two different types of building parameters, to protect one of the areas against the noise generated in the second area.

Buffer (n(d)f) aims at not only leveling off two different types of building parameters, but also to protect one of the areas against the noise emissions generated in the second area and to against a distinct function. 
Buffer (ndf) aims not only to level off two different types of building parameters, but also to protect one of the areas against the noise generated on the other area and against a distinct function.

Buffer ((npd)f) is supposed to level off two different types of building parameters, to protect one of the areas against noise and pollution generated in the second area, and to protect against a distinct function.

Buffer (nop(d)f) is supposed to level off two different types of building parameters, to protect one of the areas against noise, odor and pollution generated in the second area, and to protect against a distinct function.

\section{Conclusion}

The scope of protection of the above described buffer is diverse. First of the buffers ((d)) can be organized in the form of transition zones. They allow for a relatively smooth transition of one area to another one. The second buffer $(\mathrm{n}(\mathrm{d}))$ is similar to the first one yet the transition zone [7] should also include the acoustic protection. Since the transition zone is a strip of a relatively small size, the problem should be solved by architectures shaping the buffer (nd) will be also slightly different since due to its significant differences in building parameters it is difficult to be implemented as a transition zone. However, this fact may help protect the area against noise. In this situation you can consider the implementation of a buffer as an entirely separate area with the same basic functionality, but with averaged parameters, so that it will provide wither the protection or leveling against noise. It seems to be possible to create a buffer in the form of an area of a completely different function; however, this issue requires further analysis of such options.

Following this line of reasoning, further buffers (n(d)f) and (ndf) can be solved in a similar manner; however, being the buffer, the separate area should take into account the incompatibility of functions. It can be assumed that in certain situations, the buffer will be able to have the same basic function as one of the sites and you may find that it will be necessary to apply a completely different area or even a completely different form of the buffer.

This issue gets even more complicated as far as the buffer ((npdf)f) is concerned since this buffer aims to protect other areas against the impact of negative factors occurring in the medical services. The difficulty lies essentially in analyzing the scale of the activity. It can be assumed that the higher the degree of complexity of services, the higher the risk of $r$ factors which the remaining areas must be protected against. Problems of this buffer require a separate discussion. Also, the buffer (nop(d)f) is a specific one. The number of factors against which it must protect adjacent areas with industry is the largest. The parameters of the building - so characteristic of the industry - do not facilitate the problem. You can apply a segmental isolation of the area with an adequate number of smaller buffer spaces that will mitigate the connection between industry and any other area. The organization of an industrial land should also be carefully thought over so that objects which are less burdensome to the environment are localized at the periphery of the area. 
The abovementioned procedure presents a method of determining what a particular buffer one attempts to name. Due to a simplification, the number of buffers is relatively small. They can be called basic buffers and could be a starting point for building buffers that will relate to all factors that might appear where two areas meet. The basic buffers can be classified in the following way:

a) The transitional buffer - is to use a more stringent control over the use of land at the border zone. It can also work the other way, the controls may be relaxed in the transition area. The control can be used in the records of (architectural) building parameters, such as height, building surfaces, etc.;

b) The functional buffer - not only does it require applying relevant legal provisions but also an individual design so that it can fulfill its role properly and protect or mitigate the negative effects of the interaction between the two areas so that they form an organized Entity.

Besides them, be aware of the landscaped buffer, which was mentioned earlier.

\section{References}

[1] Rykwert, J., The Seduction of Place: The History and Future of Cities. MCK: Kraków; pp. 221-254, 2013.

[2] Barelkowski, R., Planning for sustainable development of energy infrastructure: FAST - Fast Simulation Tool, International Journal of Energy Management and Production, in print, p. 12.

[3] Norton, C. McKim., Elimination of Incompatible Uses and Structures, Law and Contemporary problems, 20(2), pp. 305-316, 1955.

[4] Jacobs, J., The health and Life of Great American Cities, Jonathan Cape: London; p. 433, 1962

[5] Barełkowski, R., The Edge of the [dis]Order, M. $7^{\text {th }}$ International Conference on Urban Regeneration and Sustainability. The Sustainable City VII. Urban Regeneration and Sustainability, eds. M. Pacetti, G. Passerini, C. A. Brebbia, G. Latini 7-9 May 2012, Wessex Institute of Technology, WIT Press, Southampton \& Boston, pp. 759-770.

[6] http://secondcityzoning.org/zones

[7] Comey, A.C., Transition Zoning. Harvard University Press: Cambridge, pp. 8-19, 1933. 